\title{
Hybrid Nanoparticles as a Novel Tool for Regulating Psychosine-Induced Neuroinflammation and Demyelination In Vitro and Ex vivo
}

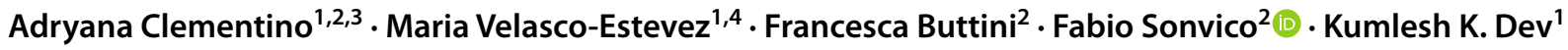

Accepted: 12 August 2021 / Published online: 3 September 2021

(c) The Author(s) 2021

\begin{abstract}
Polymeric nanoparticles are being extensively investigated as an approach for brain delivery of drugs, especially for their controlled release and targeting capacity. Nose-to-brain administration of nanoparticles, bypassing the blood brain barrier, offers a promising strategy to deliver drugs to the central nervous system. Here, we investigated the potential of hybrid nanoparticles as a therapeutic approach for demyelinating diseases, more specifically for Krabbe's disease. This rare leukodystrophy is characterized by the lack of enzyme galactosylceramidase, leading to the accumulation of toxic psychosine in glial cells causing neuroinflammation, extensive demyelination and death. We present evidence that lecithin/chitosan nanoparticles prevent damage associated with psychosine by sequestering the neurotoxic sphingolipid via physicochemical hydrophobic interactions. We showed how nanoparticles prevented the cytotoxicity caused by psychosine in cultured human astrocytes in vitro, and how the nanoparticle size and PDI augmented while the electrostatic charges of the surface decreased, suggesting a direct interaction between psychosine and the nanoparticles. Moreover, we studied the effects of nanoparticles ex vivo using mouse cerebellar organotypic cultures, observing that nanoparticles prevented the demyelination and axonal damage caused by psychosine, as well as a moderate prevention of the astrocytic death. Taken together, these results suggest that lecithin-chitosan nanoparticles are a potential novel delivery system for drugs for certain demyelinating conditions such as Krabbe's disease, due to their dual effect: not only are they an efficient platform for drug delivery, but they exert a protective effect themselves in tampering the levels of psychosine accumulation.
\end{abstract}

Keywords Brain $\cdot$ Neuroinflammation $\cdot$ Neurodegeneration $\cdot$ Nanoparticles $\cdot$ Psychosine $\cdot$ Demyelination

\section{Introduction}

Adryana Clementino and Maria Velasco-Estevez contributed equally to this work.

Fabio Sonvico

fabio.sonvico@unipr.it

Kumlesh K. Dev

devk@tcd.ie

1 Drug Development Group, School of Medicine, Trinity College Dublin, Dublin, Ireland

2 Department of Food and Drug, Università Degli Studi Di Parma, Parma, Italy

3 National Council for Scientific and Technological Development-CNPq, Brasilia, Brazil

4 H12O-CNIO Haematological Malignancies Clinical Research Unit, Centro Nacional de Investigaciones Oncológicas (CNIO), Madrid, Spain
Krabbe's disease (KD) or globoid cell leukodystrophy (GLD) is an autosomal recessive pathology caused by genetic defects in the lysosomal enzyme galactosylceramidase (GALC) [1]. This GALC deficiency results in the accumulation of the toxic intermediate metabolite galactosylsphingosine, also called psychosine (Psy) [2]. This excess of psychosine leads to its escape from lysosomes and the formation of aggregates that have cytotoxic effects by various mechanisms, such as mitochondrial dysfunction [3], S1P signaling [4], PKC, JNK and NFkB signaling [3] and inflammatory responses [5]. Ultimately, psychosine accumulation causes demyelination and almost complete loss of the oligodendrocytes in the white matter alongside glial reactivity and infiltration of multinucleated macrophages called 'globoid cells' $[1,6]$. To date, there is no current treatment for $\mathrm{KD}$, likely in part because of the yet-unknown full mechanism 
of action of Psy in the pathology, and in part because of a lack of drugs that efficiently reach the CNS and treat the pathophysiological causes of the disease, instead of treating the symptoms.

Despite the important advances in neuroscience in the past years, delivery of drugs to the CNS in an efficient manner still remains a challenge. The major reason for this is the protection that the blood brain barrier (BBB) offers, preventing the access to CNS of a huge number of drugs, especially hydrophilic and large molecular weight compounds [7, 8]. Many drugs cannot cross the BBB efficiently when delivered by the oral or intravenous route; hence, they need to be delivered via more invasive methods, such as hyperosmotic solutions disturbing the BBB permeability or infusion of drugs directly into brain structures [9]. Many of those approaches are either risky or laden with unacceptable side effects, thus leading to the need of developing new platforms for drug deliverable to exploit alternative administration routes into the CNS which are effective and non-invasive.

The development of the pharmaceutical nanotechnology in the recent years has proven nanoparticles to be an effective method for brain delivery of numerous compounds, including drugs for cancer [10], Alzheimer's disease [11], cerebral ischemia [12] and schizophrenia [13]. Previous studies in our lab have shown the potential of hybrid nanoparticles as an efficient drug delivery system for brain delivery of drugs [14]. Here, we investigated the potential use of hybrid lecithin/chitosan nanoparticles in the treatment of KD due to its protective effects in preventing the effects of psychosine both in vitro and ex vivo.

\section{Materials and Methods}

\section{Materials and Reagents}

Chitosan with deacetylation degree of $95 \%$ and viscosity 45 cP was purchased from Primex (batch TM1874, Chitoclear FG, Siglufjordur, Iceland). Lecithin Lipoid ${ }^{\circledR}$ S45 was supplied by Lipoid AD (lot \#8,030,760, Ludwigshafen, Germany). Pharmaceutical grade oil vehicles Maisine ${ }^{\mathrm{TM}} 35-1$ (batch 134,565, glyceryl monolinoleate) and Labrafac ${ }^{\mathrm{TM}}$ Lipophile WL 1349 (batch 151,738, medium chain triglycerides) were a kind gift from Gatefossé (Saint-Priest, France). Ultrapure water (Purelab Flex, ELGA-Veolia LabWater, High Wycombe, UK) was used for nanoparticles production. All other reagents used for nanoparticles characterization were of analytical grade.

Galactosylsphingosine or psychosine (Psy; sc-202781, batch \#C2015, Santa Cruz Biotechnology, Santa Cruz, CA, USA) was prepared as $10 \mathrm{mM}$ stock solution in dimethyl sulfoxide (DMSO; D8418, Sigma-Aldrich, St. Louis, MO, USA). To avoid any cytotoxic effects, organic solvent concentrations during cell treatments were maintained below $0.01 \%$.

\section{Nanoparticles Preparation and Characterization}

Lecithin/chitosan nanoparticles (LCN) were prepared by self-assembly method as previously described $[14,15]$. Briefly, $1 \% \mathrm{w} / \mathrm{v}$ chitosan was prepared by dissolving chitosan powder in $0.1 \mathrm{~N} \mathrm{HCl}$ solution overnight. The same procedure was adopted to produce nanoparticles in organic phase, consisting of $2.5 \%$ soybean lecithin dissolved in pure ethanol containing $20 \mathrm{mg}$ of glyceryl monolinoleate and medium chain triglyceride oils. Afterwards, $0.8 \mathrm{ml}$ of ethanol-oily solution of lecithin was added to $10 \mathrm{ml}$ of $0.01 \%$ $\mathrm{w} / \mathrm{v}$ of chitosan aqueous solution under constant agitation of magnetic stirring at $300 \mathrm{rpm}$ and constant temperature of $50{ }^{\circ} \mathrm{C}$. Freshly prepared LCN formulation was kept in agitation and at the same temperature for approximately $10 \mathrm{~min}$ to allow complete evaporation of ethanol. LCN particle size and polydispersity index (PDI) were then characterized by dynamic light scattering (DLS), as well as determining the nanoparticle zeta potential by electrophoresing mobility applying phase analysis light scattering (PALS) (Zetasizer Nano ZS, Malvern Instriuments Ltd., Malvern, UK). A chitosan aqueous solution $(0.01 \% \mathrm{w} / \mathrm{v})$ and lecithin dispersion $(0.2 \% \mathrm{w} / \mathrm{v})$ in water were also prepared to be used as controls, following the same manufacturing procedure, but omitting all the other LCN ingredients from the preparation.

\section{Cryo-Transmission Electro Microscopy (CryoTEM)}

For the cryo-TEM, samples of Psy $200 \mu \mathrm{M}, \mathrm{LCN} 10 \mathrm{nM}$ and Psy $200 \mu \mathrm{M}+\mathrm{LCN} 10 \mathrm{nM}$ were prepared in ultrapure water.

Briefly, $3 \mu \mathrm{l}$ of sample were applied to Quantifoil holey carbon grids (copper Multi A, Quantifoil Micro Tools GmbH, Jena, Germany). Excess fluid was blotted from the grid for $\sim 3 \mathrm{~s}$ with Whatman filter paper and then plunge frozen in liquid ethane using a home-made plunge freezer to achieve sample vitrification. Frozen samples were stored in liquid nitrogen until EM imaging. Vitrified samples were imaged using a CM200 FEG transmission EM (FEI, Eindhoven, the Netherlands) operated at $200 \mathrm{keV}$ and equipped with a F224HD $2048 \times 2048$ CCD camera (TVIPS, Gauting, Germany). EM images were acquired at 50,000 $\times$ magnification (pixel size $0.331 \mathrm{~nm})$.

\section{Human Astrocyte Culture}

Human astrocytes from foetal cerebral cortex were purchased from ScienCell Research Laboratories (Cat No. \#1800, Lot No. 9063 and 11,065; Sciencell Research Laboratories, Carlsbad, CA, USA). Ethics for obtaining human tissue were strictly adhered to by the supplier and complied 
with local, state and federal laws and regulations, and procedures for using human cells were carefully followed as previously described [16, 17]. Human astrocytes were grown in T75 culture flasks (Corning Inc., Corning, NY, USA) at $37{ }^{\circ} \mathrm{C}, 95 \%$ humidity and $5 \% \mathrm{CO}_{2}$ using Dulbecco's Modified Eagle Medium/Nutrient Mixture F-12 (DMEM/ F12) culture medium (Hyclone, SH30023, GE Healthcare Life Sciences, Chicago, IL, USA) supplemented with $10 \%$ foetal bovine serum (FBS; FB-1090, Labtech, Heathfield, UK), $1 \%$ astrocyte growth supplement (AGS; \#1852, ScienCell Research Laboratories) and 1\% penicillin/streptomycin (P/S; P4333, Sigma Aldrich). Treatments were performed in serum-free DMEM/F12 media supplemented with $1 \% \mathrm{P} / \mathrm{S}$ and cells were serum starved overnight prior treatment.

\section{Cell Viability Assay}

Cell viability was tested using the MTT assay. Briefly, cells were seeded at a density of 120,000 cells/well in a 24-well plate, grown for $48 \mathrm{~h}$ and then serum starved overnight prior treatment ( $4 \mathrm{~h}$ incubation). Dose-response curves of nanoparticles were performed to analyse their toxicity (LCN 0.01-2 nM). Similarly, Psy $(20 \mu \mathrm{M})$ was used to evaluate the efficacy of the treatment ( $\mathrm{LCN} 0.01,0.1$, $1 \mathrm{nM})$. For comparison purposes, astrocytes were further treated with Psy in presence of the main LCN components, i.e. chitosan and lecithin, at concentrations matching those present in $1 \mathrm{nM} \mathrm{LCN}$. Prior to MTT incubation after treatment, representative images of cells in all treatment studies were taken using a CKX41 Olympus inverted microscope (Mason Technologies, Dublin, Ireland). Cells were incubated with $1 \mathrm{mg} / \mathrm{ml}$ MTT reagent (M6494, Invitrogen, Thermo Fisher Scientific, Waltham, MA, USA) for $3 \mathrm{~h}$ at $37{ }^{\circ} \mathrm{C}$, following the addition of DMSO and absorbance read at $540 \mathrm{~nm}$ using an Epoch microplate spectrophotometer (BioTek, Winooski, VE, USA). Absorbance values were directly correlated with the cellular viability and values were calculated as percentage of control.

\section{Immunocytochemistry}

Immunocytochemistry of cultured human astrocytes was performed as previously reported $[16,18]$. Briefly, cells were seeded and grown in sterile glass coverslips on 24-well plates and treated as described above ( $4 \mathrm{~h}$ incubation with Psy $20 \mu \mathrm{M}$ or Psy $20 \mu \mathrm{M}+\mathrm{LCN} 1 \mathrm{nM}$; untreated astrocytes were used as controls). After treatment, cells were washed and fixed in 4\% PFA for 5 min on ice. Cells were then washed in PBS and permeabilized with $0.1 \%$ Triton-X100 in PBS for $5 \mathrm{~min}$, room temperature (RT). Afterwards, cells were blocked with $1 \%$ BSA in PBS $+0.1 \%$ Triton-X100, at $4{ }^{\circ} \mathrm{C}$ overnight. Following blocking, samples were incubated with primary antibody mouse anti-vimentin (sc373717,
Santa Cruz Biotechnology; dilution 1:800) and with secondary antibody Dylight 549 anti-mouse (\#715-505-020, Jackson ImmunoResearch, Ely, UK; dilution 1:1000). Cells were when washed and incubated with DAPI (\#62,248, Thermo Fisher Scientific; dilution 1:500) for nuclear staining and mounted on a microscope slide using antifade reagent (S36936, Thermo Fisher Scientific). Coverslips were sealed and samples were stored at $4{ }^{\circ} \mathrm{C}$ until imaged using an Olympus Bx51 upright fluorescence microscope at $20 \times$ magnitude. Image acquisition settings were kept constant across all treatments. Image analysis of fluorescence was made using the ImageJ software version 1.52a (https:// imagej.nih.gov/ij).

\section{Enzyme-Linked Immunosorbent Assay (ELISA)}

Human cytokine IL-6 supernatant levels were measured using an IL-6 ELISA Kit (DY206, R\&D Systems, Minneapolis, MN, USA), following manufacturer's instructions. Briefly, 96-well plates of maxiabsorbant (\#442,404, SigmaAldrich) were coated with the capture antibody diluted in PBS, overnight at $4{ }^{\circ} \mathrm{C}$. Plates were washed in $0.05 \%$ PBS/Tween-20 and blocked at RT for a minimum of $1 \mathrm{~h}$. Plates were then washed again and incubated with samples and standards for $2 \mathrm{~h}$ at RT. After following washes, plates were incubated with detection antibody for further $2 \mathrm{~h}$ at RT and washed again before incubation with streptavidin-HRP solution for $20 \mathrm{~min}$ in the dark. ELISA was developed using substrate solution (DY999, R\&D Systems). The reaction was stopped by adding $2 \mathrm{~N} \mathrm{H}_{2} \mathrm{SO}_{4}$ and absorbance was read at $450 \mathrm{~nm}$ with wavelength correction at $570 \mathrm{~nm}$.

\section{Cerebellar Organotypic Slice Culture}

Mouse cerebellar organotypic slice culture was performed using postnatal day $10 \mathrm{C} 57 \mathrm{bl} / 6$ mice (P10) provided by BioResources Unit, Trinity College Dublin (Ireland). All tissue was isolated in accordance with EU regulations and internal protocols approved by Trinity College Dublin ethical committee. Mice were sacrificed by decapitation, the skull removed and the cerebellum separated from hindbrain. Cerebellum was cut into $400 \mu \mathrm{m}$ parasagittal sections using a McIlwan tissue chopper. Tissue was placed into Opti-MEM (\#31,985, Gibco, Thermo Fisher Scientific) and separated into individual slices under a dissection microscope. Five slices were placed per cell culture insert (PICMORG50, Merk Millipore, Burlington, MA, USA) and grown at $35.5{ }^{\circ} \mathrm{C}, 95 \%$ humidity and $5 \% \mathrm{CO}_{2}$. Slices were grown for the first 4 days in media containing 50\% Opti-MEM, 25\% Hank's buffered salt solution (HBSS; \#14,025-050, Gibco, Thermo Fisher Scientific) and 25\% heat-inactivated horse serum (\#26,050-088, Gibco, Thermo Fisher Scientific) supplemented with $2 \mathrm{mM}$ Glutamax (\#35,050, Gibco, Thermo 
A

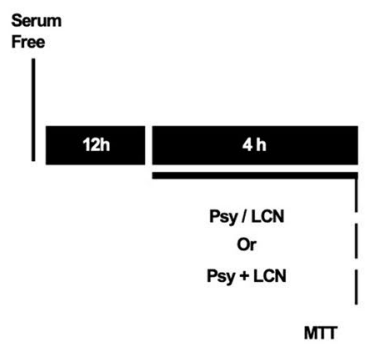

C
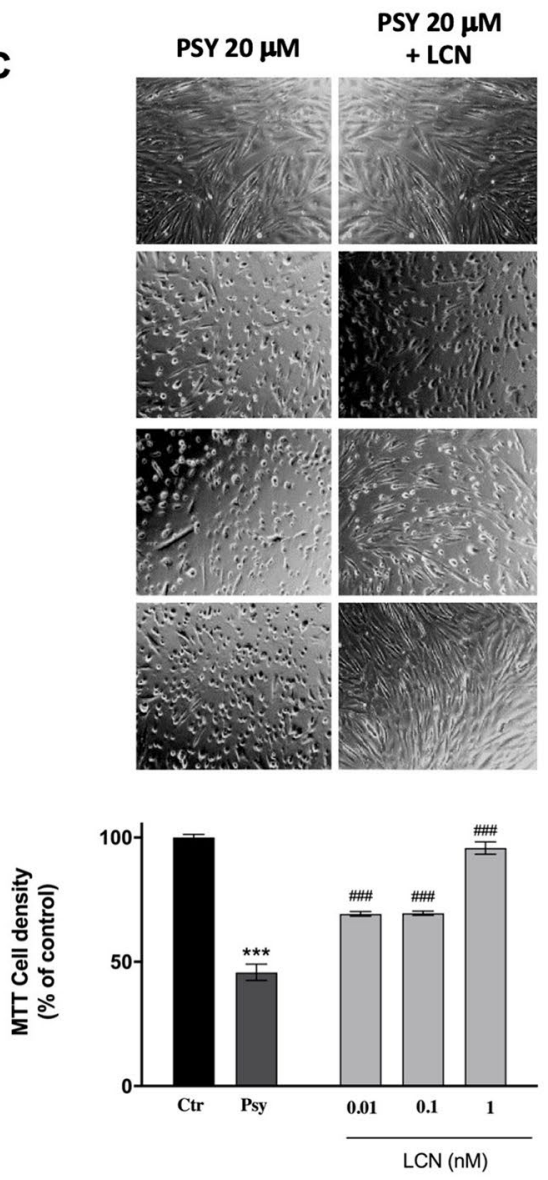

E

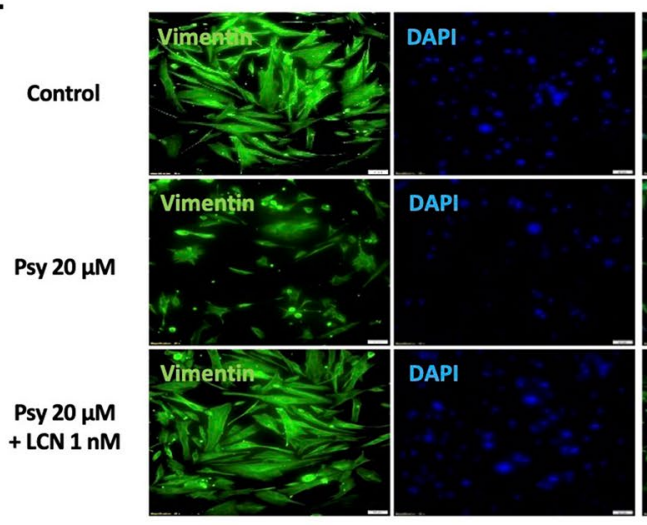

B

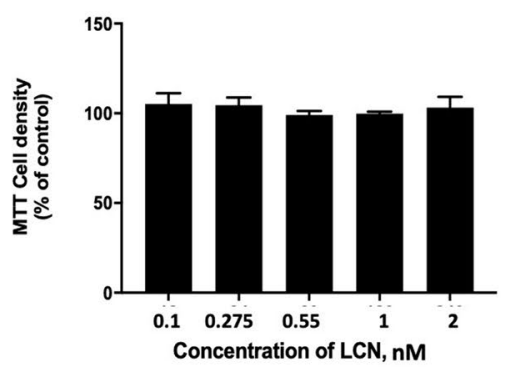

D
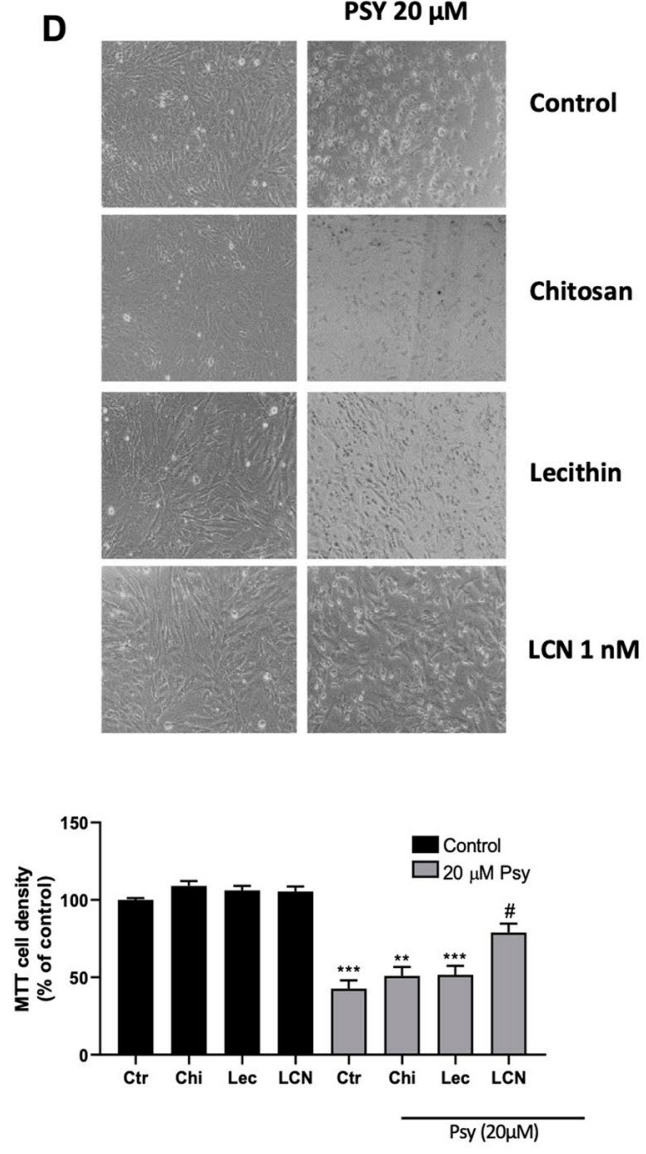
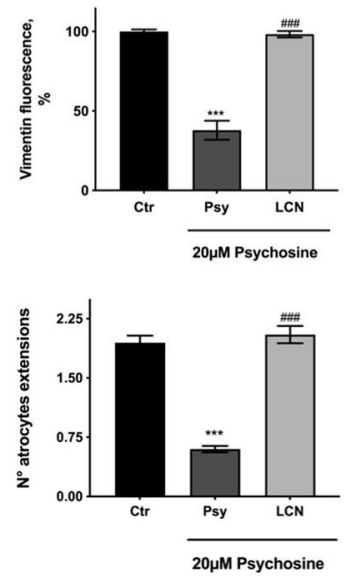

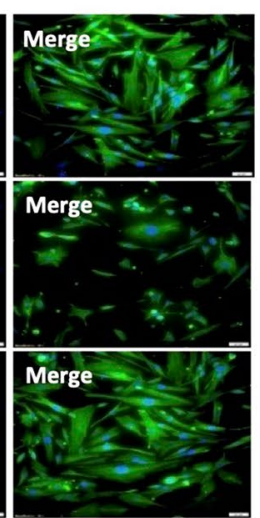

$0.01 \mathrm{nM}$

LCN

$0.1 \mathrm{nM}$

LCN

$1 \mathrm{nM}$

LCN 
४Fig. 1 Nanoparticles (but not the individual components) protect from Psy-induced astrocytic cell death. A Experimental diagram of treatments and analysis. Briefly, human astrocytes were serum starved overnight and then treated for $4 \mathrm{~h}$ with psychosine $(20 \mu \mathrm{M}$, Psy), lecithin/chitosan nanoparticles (LCN, 0.1-2 nM) or the combination of Psy $(20 \mu \mathrm{M})$ and increasing concentrations of LCN (0.01, 0.1 and $1 \mathrm{nM})$. B LCN nanoparticles did not show cytotoxicity in human astrocytes at any of the concentration used, as measured by MTT assay and having untreated astrocytes as control. C Light microscope images and MTT assay showed LCN protection against $20 \mu \mathrm{M}$ Psy-induced cell death, in a dose-dependent manner, tested at $0.01 \mathrm{nM}, 0.1 \mathrm{nM}$ and $1 \mathrm{nM}$. D However, light microscopy images and MTT assay showed that lecithin or chitosan on their own failed to protect astrocytes from Psy effects. E Fluorescent images at $20 \times$ magnification of human astrocytes labelled for vimentin (green) and DAPI (blue) showing a protective effect of LCN from the Psyinduced toxic effects, measured by intensity of vimentin expression and number of astrocytic extensions. Scale bar $=50 \mu \mathrm{m}$. Fluorescence analysis was performed using ImageJ software. Data shown as mean \pm SEM. Statistical analysis performed using one-way ANOVA following Holm-Sidak's post hoc test. $* * * p<0.001$ compared to control; ${ }^{\# \# \# p} p<0.001$ compared to Psy. $N=8$ for cell viability assays; $N=4$ for fluorescence analysis

Fisher Scientific), $28 \mathrm{mM}$ D-Glucose (G8769, SigmaAldrich), 1\% P/S and HEPES (\#15,630-056, Gibco, Thermo Fisher Scientific), changing the media at day in vitro 1 (DIV1) and DIV4. At DIV7, media was changed to a serumfree media containing 96\% Neurobasal-A (\#10,888-022, Gibco, Thermo Fisher Scientific) and 2\% B-27 supplement (\#17,504-044, Gibco, Thermo Fisher Scientific) supplemented with $1 \%$ P/S, $28 \mathrm{mM}$ D-Glucose, $2 \mathrm{mM}$ Glutamax and $10 \mathrm{mM}$ HEPES. Media was changed again at DIV10 and treatment was performed at DIV12.

\section{Immunofluorescence}

After treatment of organotypic cultures, fixation was done by increasing concentration of PFA $(1 \%, 2 \%, 3 \%$ and $4 \%)$ for $5 \mathrm{~min}$ each. Blocking and permeabilization were performed overnight at $4{ }^{\circ} \mathrm{C}$ in blocking solution $10 \%$ bovine serum albumin (BSA; \#1,073,508,600, SigmaAldrich) in PBS $+0.5 \%$ Triton-X100 (Tx). Primary antibodies were diluted in $2 \% \mathrm{BSA}$ in PBS $+0.1 \% \mathrm{Tx}$, incubating for $48 \mathrm{~h}$ at $4{ }^{\circ} \mathrm{C}$. Slices were then washed in PBS 5 times for $5 \mathrm{~min}$ and incubated with secondary antibodies diluted in $2 \%$ $\mathrm{BSA}+0.1 \%$ Tx overnight at $4{ }^{\circ} \mathrm{C}$. Slices were then washed again and mounted on a slide microscope using SlowFade ${ }^{\circledR}$ Gold antifade reagent (S36936, Life Technologies, Thermo Fischer Scientific).

\section{Confocal Microscopy and Image Analysis}

Imaging of organotypic cultures was performed using a Leica SP8 confocal microscope (Leica Microsystems, Wetzlar, Germany) in TBSI, Trinity College Dublin. Five slices per condition were grown and an average of 25-3 images per condition were captured at $20 \times$ magnification. The images were exported as 8-bit.tif files for analysis using the software package ImageJ by FIJI (https://imagej.net/Fiji). Intensity values were normalized to the average of control for each experiment and each marker. To analyse the expression of SMI-32 in the white matter tracts (WMT), the software package Imaris ${ }^{\circledR}$ (http://www.bitplane.com/imaris/imaris) was used. Briefly, the WMT on each image were selected and the area of SMI-32 immunoreactivity was measured.

\section{Statistical Analysis}

All statistical analysis was performed using GraphPad Prism 8 (GraphPad ${ }^{\circledR}$ Software, San Diego, CA, USA). Assessment of the normality of the data was carried out by column statistics with D'Agostino analysis before any further statistical test was performed. To analyse cell viability via MTT assay, immunocytochemistry images and IL-6 cytokine release under multiple conditions, repeated measures one-way ANOVA test was performed, as data in every experiment was matched and with Gaussian distribution. Holm-Sidak multiple comparisons post hoc test was run in conjunction with one-way ANOVA and all groups were compared between themselves. Similarly, to analyse fluorescence intensity levels in organotypic slice cultures, repeated measures one-way ANOVA following Sidak's post hoc test was performed. $P$ values $<0.05$ were considered statistically significant for all experiments. Data is presented as mean \pm standard error of the mean (SEM). Further details of the statistical analysis performed are given in each figure legend and the results section.

\section{Results}

\section{Hybrid Lecithin/Chitosan Nanoparticles Protect Human Astrocytes from Psychosine-Induced Cytotoxicity In Vitro}

Administering drugs encapsulated into polymeric nanoparticles has proven to be an efficient way of targeting the CNS and improving their pharmacokinetics. Here, we developed and studied hybrid LCN which can potentially be used to encapsulate drugs for brain delivery after intranasal administration. Following a simple and reproducible manufacturing technique which exploits the electrostatic self-assembly of phospholipids and polysaccharides, $\mathrm{LCN}$ were obtained with relatively small particle size $(234.2 \pm 8.8 \mathrm{~nm})$, presenting narrow particle size distribution (PDI $0.094 \pm 0.009$ ) and surface charge sufficiently strong to provide good physical stability (zeta potential of $+40.21 \pm 1.09 \mathrm{mV}$ ). Here, we first tested the toxicity of blank LCN at increasing concentrations in cultured human astrocytes. LCN were incubated 
for $6 \mathrm{~h}$ at concentrations ranging from 0.1 to $2 \mathrm{nM}$, and cell viability of astrocytes was measured by MTT assay. We observed that none of the LCN concentrations used evidenced significant cytotoxic effects on the astrocytes (Fig. 1B). We then tested the potential protection of these LCN against psychosine toxicity. Psychosine is a toxin that accumulates in the brains of patients affected by Krabbe's disease, due to the deficit of the lysosomal enzyme galactocerebrosidase [6]. Previous studies in our lab have shown that Psy induces astrocytic cell death in a concentration and time-dependent manner $[4,19]$. Thus, astrocytes were incubated with $20 \mu \mathrm{M}$ of Psy in the presence or absence of LCN at concentrations ranging from 0.01 to $1 \mathrm{nM}$. It was observed that LCN efficiently protect against Psy-induced cytotoxicity, with the highest protection observed at $1 \mathrm{nM} \mathrm{LCN}$, presenting cell densities similar to control (LCN $0.01 \mathrm{nM}$ : $69.34 \% \pm 2.98 \%$; LCN $0.1 \mathrm{nM}: 69.59 \% \pm 2.8 \%$; LCN $1 \mathrm{nM}: 95.80 \% \pm 4.86 \%$ of control vs Psy: $45.73 \% \pm 6.63 \%$ ) (Fig. 1C). In order to better understand the mechanism of action through which LCN protect from Psy-induced toxicity, we analysed the effects on cell viability of the individual components of $\mathrm{LCN}$, i.e. lecithin and chitosan, at concentrations matching those present in LCN $1 \mathrm{nM}$. Interestingly, the individual components of the nanoparticles had no protective effects on astrocytes (chitosan: 50.89\% \pm 5.79 ; lecithin: $51.74 \% \pm 5.69$ vs Psy: $42.71 \% \pm 5.30 \%$ ) (Fig. 1D). To further assess the protective effects of LCN, we analysed the effects of $\mathrm{LCN}$ on the expression of the astrocytic cytoskeleton marker vimentin by immunofluorescence. Treatment of human astrocytes with $20 \mu \mathrm{M}$ Psy caused a decrease in the expression of vimentin in astrocytes and in the number of astrocytic projections (Psy: $37.86 \pm 6.02 \%$ vimentin expression compared to control; $0.24 \pm 0.08$ astrocytic projections

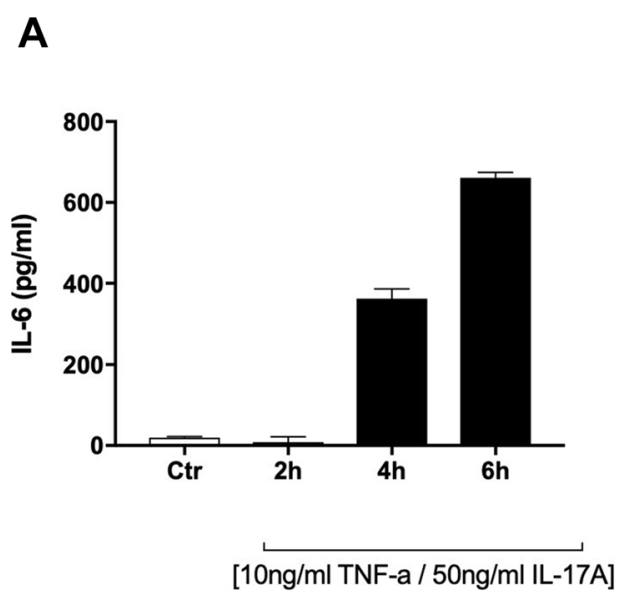

Fig. 2 Nanoparticles effects are not mediated via inflammatory mechanism. A TNF $\alpha$ and IL-17a increased the level of IL-6 release in human astrocytes. Human astrocytes were seeded at $3.9 \times 10^{5}$ cells $/ \mathrm{ml}$ confluency and grown for $48 \mathrm{~h}$. Cells were then treated with $10 \mathrm{ng} /$ $\mathrm{ml} \mathrm{TNF}-\alpha$ and $50 \mathrm{ng} / \mathrm{ml} \mathrm{IL-17a}$ for $2 \mathrm{~h}, 4 \mathrm{~h}$ and $6 \mathrm{~h}$. Graph shows vs $2.14 \pm 0.16$ for control). This decrease, however, was prevented by $1 \mathrm{nM}$ LCN co-treatment, where values of vimentin fluorescence intensity and number of projections were similar to control conditions $(98.30 \pm 2.4 \%$ vimentin expression and $2.05 \pm 0.11$ astrocytic projections, respectively) (Fig. 1E).

\section{Nanoparticles Effects are not Mediated via Inflammatory Mechanisms}

Previous studies in our lab have shown that human astrocytes in vitro express IL-17A receptor and that stimulation of this receptor causes the release of proinflammatory cytokine IL-6, which is synergistically increased by the addition of TNF $\alpha$ [18], where maximum release of IL-6 is observed at concentrations of $10 \mathrm{ng} / \mathrm{ml} \mathrm{TNF} \alpha$ plus $50 \mathrm{ng} /$ $\mathrm{ml} \mathrm{IL}-17 \mathrm{a}$ for $18 \mathrm{~h}$ sphinganine glycosylated derivatives. Here, we confirm this proinflammatory effect at 2,4 and $6 \mathrm{~h}$, showing that TNF $\alpha+\mathrm{IL}-17 \mathrm{a}$ lead to the release of IL- 6 after $4 \mathrm{~h}$ of treatment, with maximum release at $6 \mathrm{~h}$ treatment $(661.3 \pm 22.6 \mathrm{pg} / \mathrm{ml})$ (Fig. 2A). To analyse whether the protective effects of LCN were restricted to preventing the cytotoxic effects of Psy or they could protect against proinflammatory stimuli as well, human astrocytes were stimulated with $10 \mathrm{ng} / \mathrm{ml} \mathrm{TNF} \alpha$ plus $50 \mathrm{ng} / \mathrm{ml} \mathrm{IL-17a}$ in the absence or presence of $1 \mathrm{nM} \mathrm{LCN}$ for $6 \mathrm{~h}$. It was observed that while LCN did not cause any release of IL- 6 on their own $(45.0 \pm 7.7 \mathrm{pg} / \mathrm{ml}$ vs control conditions of $52.17 \pm 14.67 \mathrm{pg} /$ $\mathrm{ml}$ ), they failed to prevent the release of IL- 6 caused by $\mathrm{TNF} \alpha+\mathrm{IL}-17 \mathrm{a}(648.0 \pm 23.5 \mathrm{pg} / \mathrm{ml}$ vs $668.2 \pm 89.5 \mathrm{~g} / \mathrm{ml})$ (Fig. 2B). This suggests that the protective effects of LCN

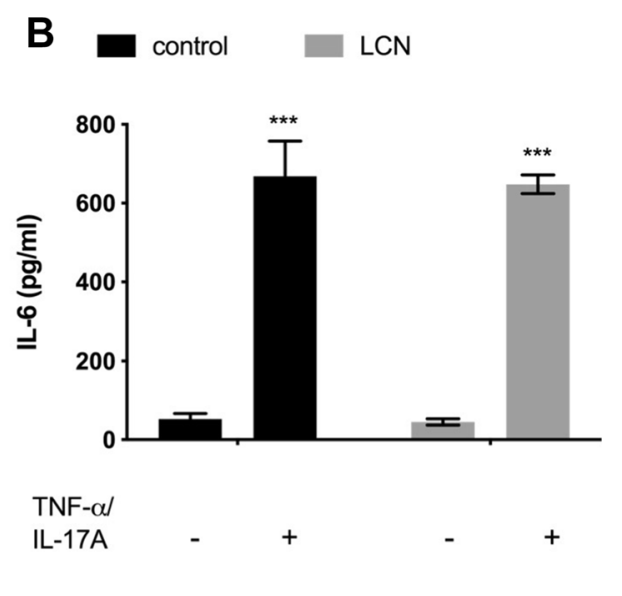

the secretion of IL-6 over time under cytokine stimuli. B LCN nanoparticles did not affect IL-6 release in human astrocytes after TNF $\alpha /$ IL-17a stimulation. Data shown as mean \pm SEM. Repeated measures one-way ANOVA following Holm-Sidak's post hoc test was performed. ${ }^{* * *} p<0.001$ compared to negative control. $N=6$ 
are restricted to preventing the damage to astrocytes induced by Psy but not those caused by the inflammatory responses.

\section{LCN-Protective Effect is Based on their Structure}

Having observed that assembled LCN protected against cytotoxicity induced by Psy but had no effect on preventing cytokine release caused by TNF $\alpha+$ IL-17a, we hypothesize that this effect could be related on nanoparticles direct interaction with Psy driven by the particle properties. Thus, the physicochemical properties of LCN were analysed using DLS measurements, reporting particle diameter, particle size distribution and zeta potential of LCN at $0.01,0.1$ and $1 \mathrm{nM}$ in the presence of $20 \mu \mathrm{M}$ of Psy. Interestingly, we observed that in the presence of Psy, LCN were found to have a concentrationdependent increase in their average diameter, compared to LCN alone (Psy+LCN $0.01 \mathrm{nM}$ : 733.1 $\pm 58.3 \mathrm{~nm}$; Psy +LCN $0.1 \mathrm{nM}: 1046 \pm 60.4 \mathrm{~nm}$; Psy+LCN $1 \mathrm{nM}: 1191 \pm 30.2 \mathrm{~nm}$ vs control LCN: $234.2 \pm 8.8 \mathrm{~nm}$ ), while at the same time they decreased their surface charge $($ Psy $+\mathrm{LCN}$ $0.01 \mathrm{nM}:+3.20 \pm 0.87 \mathrm{mV} ;$ Psy+LCN $0.1 \mathrm{nM}:+1.09 \pm 0.04 \mathrm{mV}$; Psy $+\mathrm{LCN} 1 \mathrm{nM}:+0.69 \pm 0.02 \mathrm{mV}$ ). Furthermore, PDI of LCN increased in the presence of Psy (Psy $+\mathrm{LCN} 0.01 \mathrm{nM}$ : $0.684 \pm 0.082$; Psy + LCN $0.1 \mathrm{nM}: 0.321 \pm 0.056$; Psy $+\mathrm{LCN}$ $1 \mathrm{nM}: 0.218 \pm 0.048 \mathrm{~nm}$ vs control LCN: $0.094 \pm 0.009$ ) (Fig. 3) indicating a broad particle size distribution. These results suggest that LCN physical interactions with Psy may be the responsible for the protective effects observed in vitro on cultured astrocytes. Since, however, also Psy by itself at this concentration forms micelles with a low positive surface charge and aggregates $(294.5 \pm 33.5 \mathrm{~nm}$, PDI $0.262 \pm 0.072$ and zeta potential $9.9 \pm 0.2 \mathrm{mV}$; see also Fig. 3), as reported also by others [20], we decided to proceed with the direct observation of the samples by cryoTEM. In the cryoTEM images, it appears clear that Psy forms small structures in the range of $35-50 \mathrm{~nm}$ attributed to micelles (Fig. 4A) but also larger agglomerates (120-200 nm) with a more complex inner structure (Fig. 4B). LCN nanoparticles, on the contrary, appear as spherical particles with a wide particle size distrbution (70-200 nm, Fig. 4C) often associated in groups of multiple particles (Fig. 4D). When the two materials were mixed, it was impossible to identify Psy micelles or agglomerates, while the LCN structures were still present but with an important increase of larger structures of around 200-300 nm (Fig. 4E). Smaller structures bound to the surface of larger particles were also observed (Fig. 4F).

\section{Psy-Induced Demyelination is Prevented by LCN in Organotypic Slice Cultures}

Psy accumulation in Krabbe's disease leads to oligodendrocyte death and demyelination [2]. We have previously demonstrated in our lab that Psy also induces demyelination in

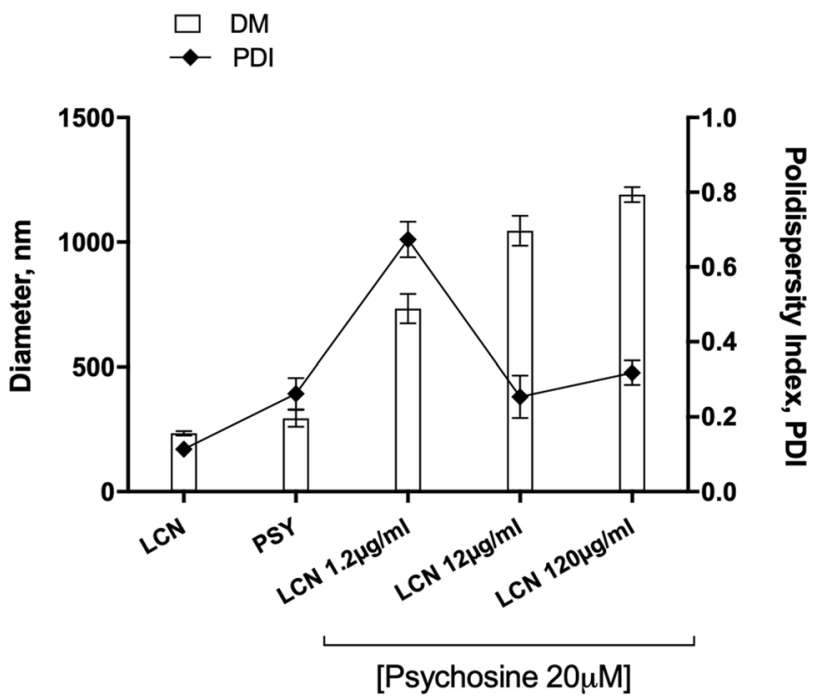

Fig. 3 Evidence of mechanical effect of LCN nanoparticles sequestering Psy. Hydrodynamic particle diameter and particles size distribution of LCN nanoparticles combined with Psy were investigated by DLS measurements. Particles diameter increased proportionally with the crescent concentration of LCN plus $20 \mu \mathrm{M}$ Psy. Data shown as mean \pm SEM. $N=3$ with six runs per analysis

mouse cerebellar organotypic slice culture $[4,19]$ and that this system can be used as an ex vivo model of demyelinating diseases to study pharmacological effects on demyelination, neuronal and glial cell effects. Organotypic slices from P10 C57B1/6 mice were grown in vitro for 12 days (DIV12), when they were treated with in the presence or absence of $100 \mathrm{nM}$ Psy with or without $1 \mathrm{nM} \mathrm{LCN} \mathrm{(Fig.} \mathrm{5A).} \mathrm{As} \mathrm{pre-}$ viously observed, Psy treatment induced demyelination in slices, reducing the levels of expression of both myelin markers MOG $\left(53.39 \% \pm 5.92 \%\right.$ of control values, ${ }^{* * *} p<0.001$, RM one-way ANOVA Sidak's multiple comparisons test) (Fig. 5B, C) and MBP $(51.35 \% \pm 7.00 \%$ of control, $* * * p<0.001$ ) (Fig. 5D, E). However, this demyelinating effect was prevented with co-treatment of Psy plus $1 \mathrm{nM}$

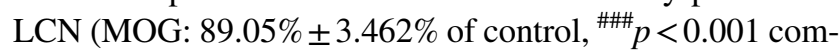
pared to Psy; MBP: $83.27 \% \pm 7.19 \%$ of control, ${ }^{\# \#} p<0.01$ compared to Psy) (Fig. 5B-E). These results corroborate the protective effect of LCN preventing the cytotoxic effects of Psy observed in astrocytes in vitro.

\section{LCN Prevents Psy-Induced Axonal Injury}

We and others have previously reported axonal injury under demyelinating conditions [19, 21, 22]. Thus, we examined the effects of LCN in the control of axonal damage induced by Psy, by measuring the expression of SMI-32, a non-phosphorylated epitope of NFH [23]. In accordance with our previous studies, Psy induced axonal damage related to demyelination, as observed in the higher 
Fig. 4 CryoTEM images of Psy, LCN and their combination. Representative cryoTEM images obtained for dilutions in ultrapure water of $\mathbf{A}, \mathbf{B}$ Psy $(200 \mu \mathrm{M}) ; \mathbf{C}, \mathbf{D}$ LCN nanoparticles $(10 \mathrm{nM})$ and $\mathbf{E}, \mathbf{F}$ their combination (Psy $200 \mu \mathrm{M}+\mathrm{LCN} 10 \mathrm{nM})$. Scale bars $=100 \mathrm{~nm}$
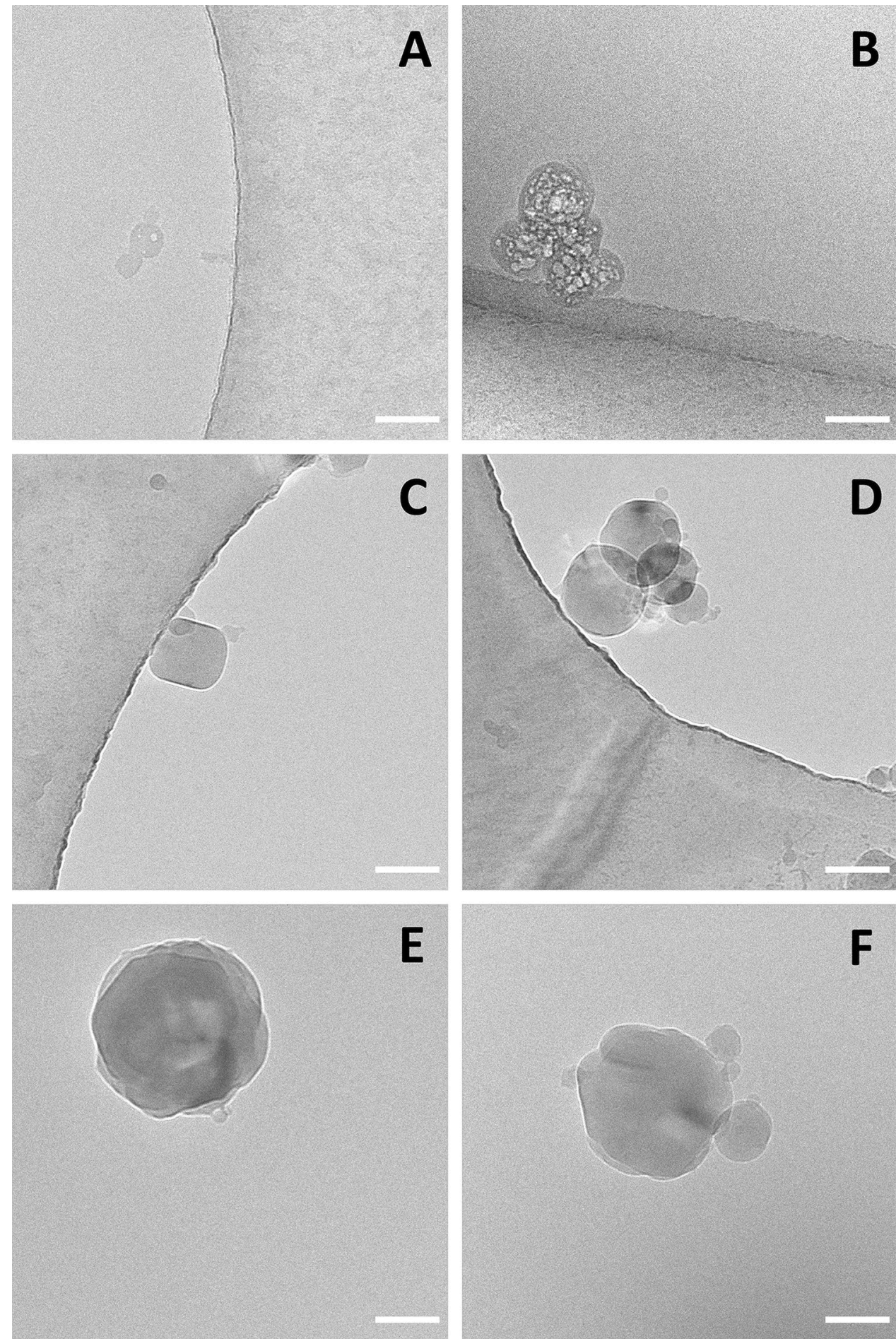

expression of SMI-32 in the axonal tracts of the arbor vitae $(157.3 \% \pm 5.66 \%$ of control values, $* * p<0.01$, RM one-way ANOVA, Sidak's multiple comparisons test). However, LCN treatment in organotypic slices not only prevented demyelination but also prevented the axonal expression of SMI-32 in the myelinated tracts as well $\left(103.5 \% \pm 5.84 \%,{ }^{\#} p<0.05\right.$ compared to Psy) (Fig. 6). This suggests that LCN prevents the Psy-induced demyelination thus preventing the associated axonal damage on the cerebellar Purkinje neurons. 
A

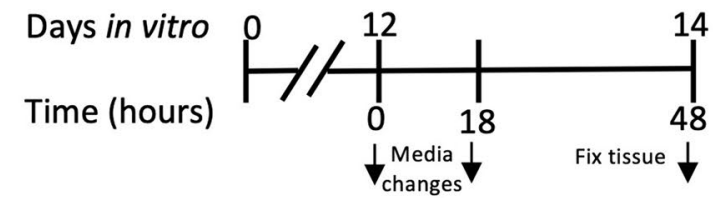

1. Control (fresh media)

2. $\operatorname{LCN}(1 \mathrm{nM})$

3. Psychosine (100nM)

4. Psychosine + LCN

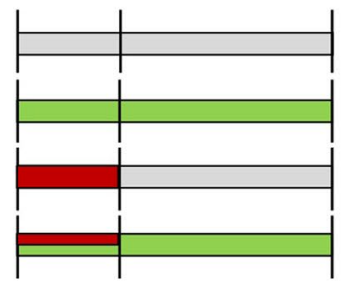

C

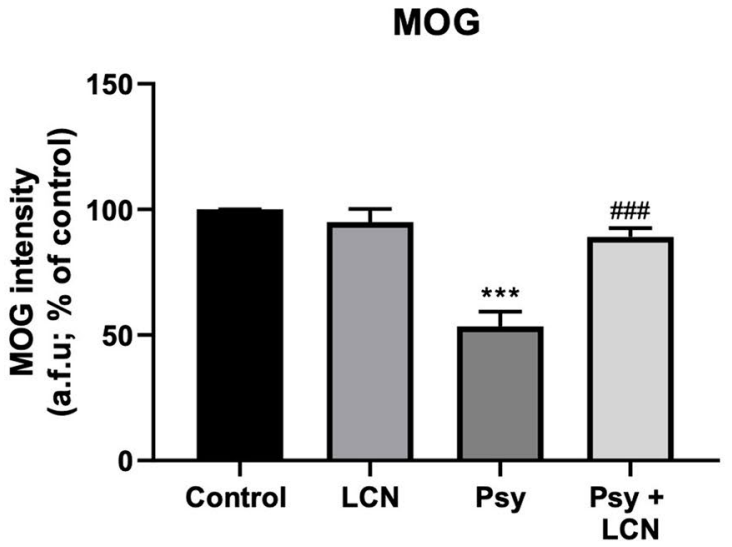

$\mathbf{E}$

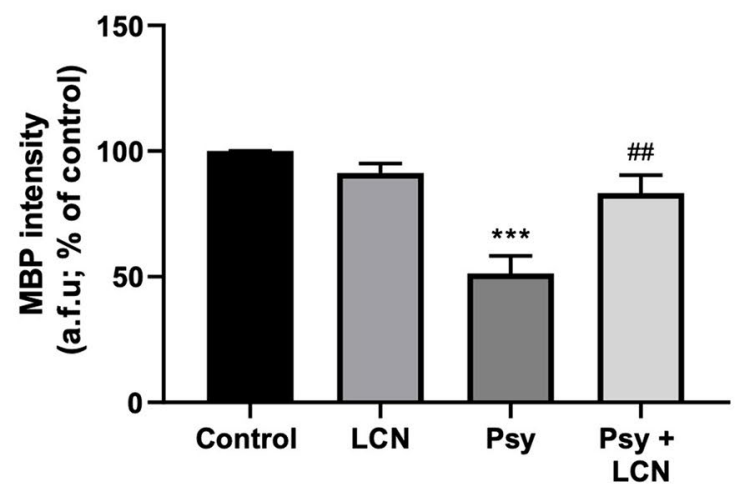

Fig. 5 LCN protect against Psy-induced demyelination. Cerebellar organotypic slice cultures were used as an ex vivo model. A Schematic treatment protocol, showing that cerebellar slices were obtained from P10 mice and cultures in vitro for 12 days. At DIV12, treatment was added and replaced to fresh change after $18 \mathrm{~h}$, until $48 \mathrm{~h}$ treatment. Slices were then fixed, blocked and prepared for staining. B, C
B
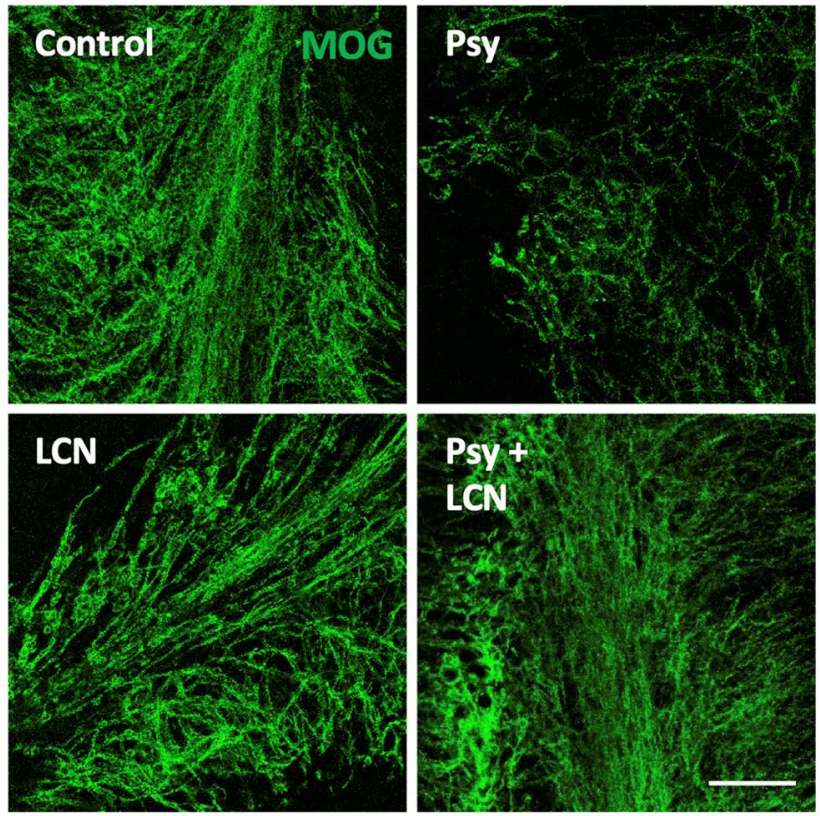

D
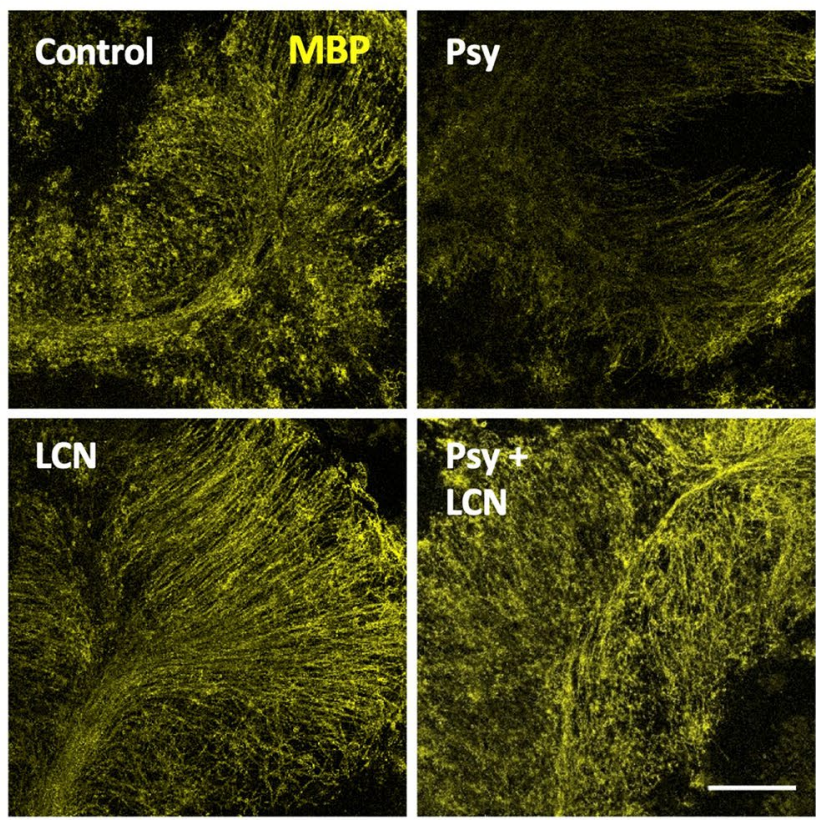

Analysis of MOG levels show a decrease in the myelin marker under psychosine treatment, which was prevented by LCN. D, E Similar results were obtained for MBP levels. Scale bar $=100 \mu \mathrm{m}$. Data is shown as mean \pm SEM. Repeated measures one-way ANOVA following Holm-Sidak's post hoc test was performed. $N=8$ 


\section{A}
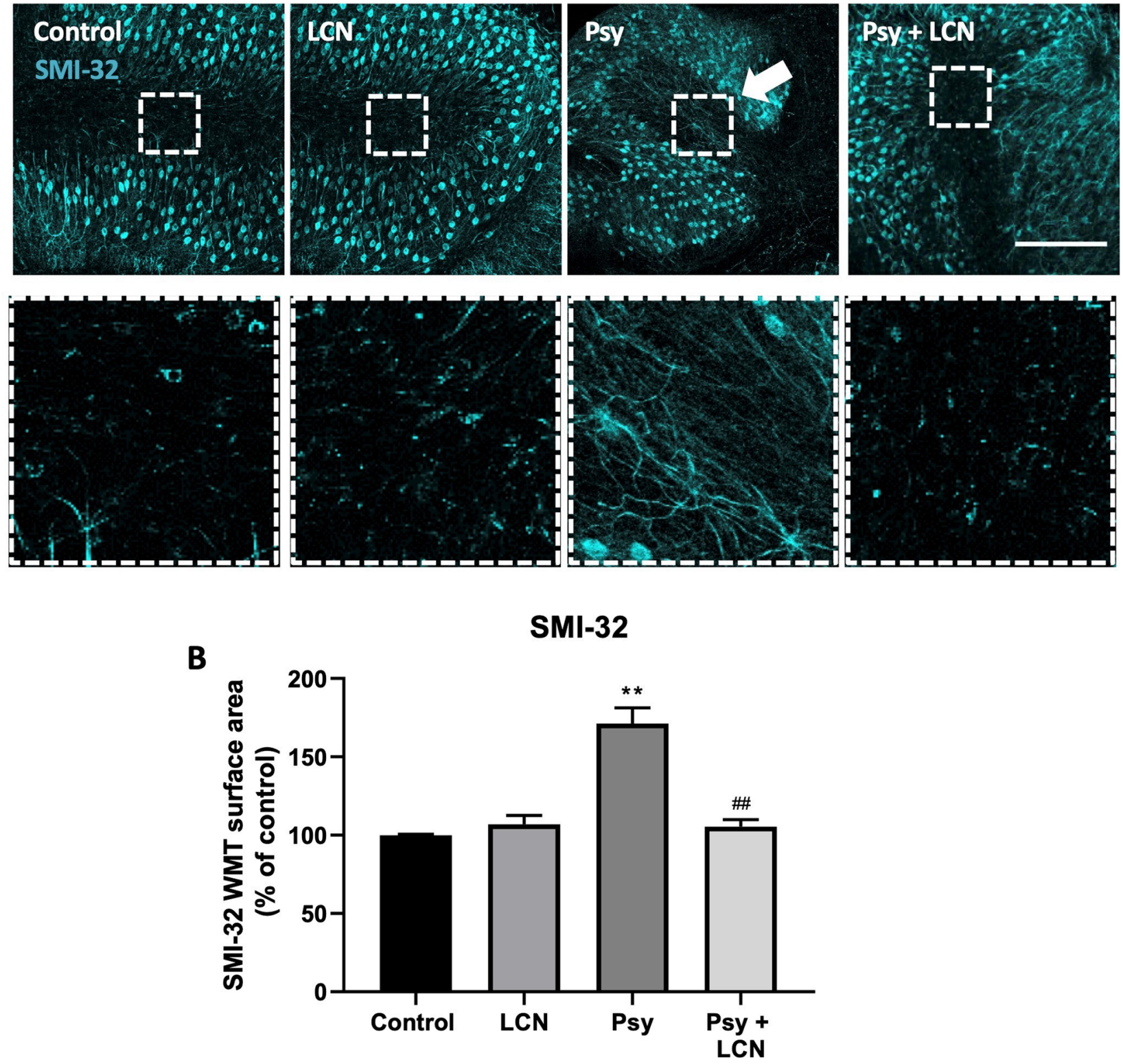

Fig. 6 LCN prevented Psy-mediated axonal injury in cerebellar slices. A Immunofluorescence analysis of neuronal SMI-32 marker in cerebellar slices. B Analysis of the surface expression reveals an increase of axonal SMI-32 in the white matter tracts under psychosine treat-

\section{Astrocytic Death Induced by Psy was not Fully Prevented by LCN Ex Vivo}

As we observed a protective effect of LCN preventing the cytotoxicity of Psy in human astrocytes in vitro, we then examined the expression of astrocytic maker GFAP and astrocytic and early astrocytic marker vimentin. Similar to what observed in previous studies in our lab, Psy induced astrocytic death measured by a decrease in the expression of GFAP $(57.68 \% \pm 7.71 \%$ of control values, $* p<0.05$, RM one-way ment. However, this expression was prevented in co-treatment with LCN. Scale bar $=100 \mu \mathrm{m}$. Data shown as mean \pm SEM. Repeated measures one-way ANOVA following Holm-Sidak's post hoc test was performed. $N=6$

ANOVA with Sidak's multiple comparisons test) (Fig. 7A, B) and a non-significant tendency to decrease in the expression of vimentin $(79.15 \% \pm 8.49 \%$, of control values, n.s.) (Fig. 7C, D). In contrast with what observed in our in vitro experiments (Fig. 1), LCN treatment did not prevent the Psy-induced astrocytic death ex vivo to control levels, although it caused a nonsignificant increase in the expression of GFAP compared to Psy-treated levels $(83.14 \% \pm 2.57 \%, * p<0.05$ compared to control, n.s. compared to Psy) (Fig. 7A, B). When observing the effects on microglia by measuring the expression of 
A
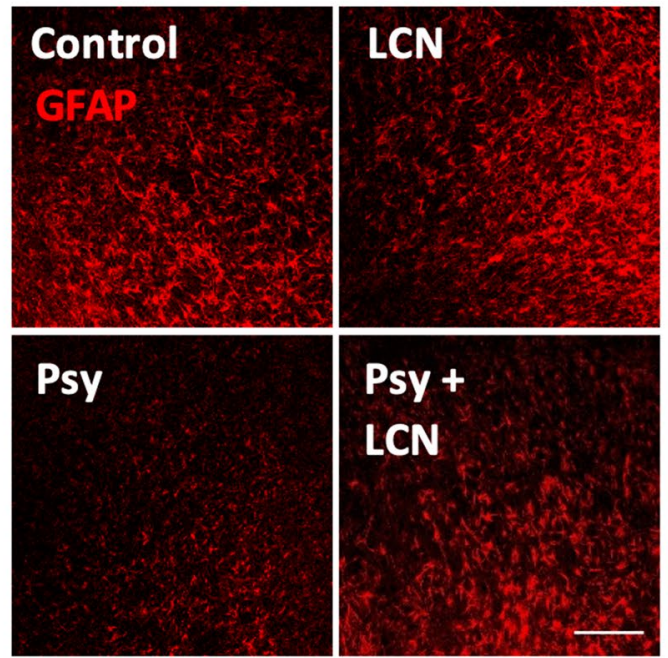

C
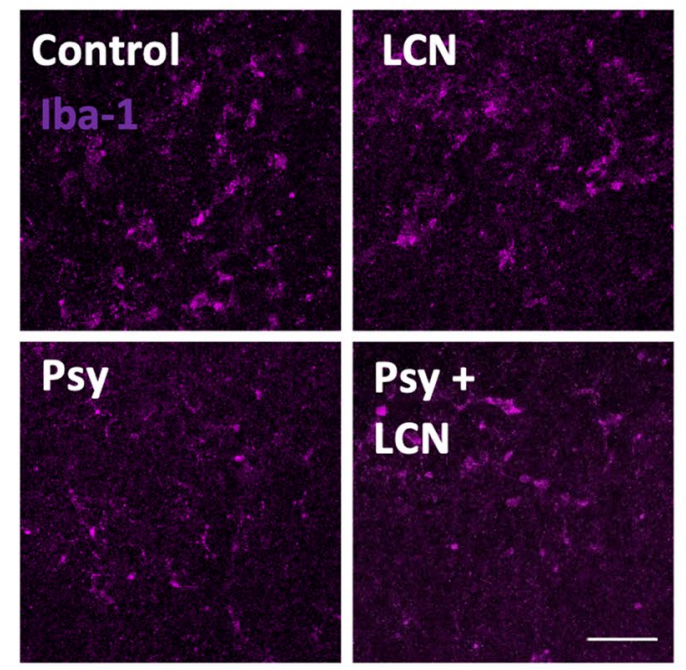

Fig. 7 Psy-induced astrocytic death was not fully prevented by LCN in cerebellar slices. A, B Immunofluorescence of astrocytic GFAP marker showed a significant decrease of GFAP levels in Psy-treated slices, while LCN had little effect reverting this loss. C, D Astrocytic and early glia marker, vimentin, showed no apparent difference in

the microglial marker Iba-1, none of the treatments had any apparent effect on the levels of expression of this microglial marker (Fig. 7E, F).

\section{Discussion}

\section{Summary of Findings}

Krabbe's disease is a fatal leukodystrophy with no current curative treatment. Therefore, a need for new drugs and
B
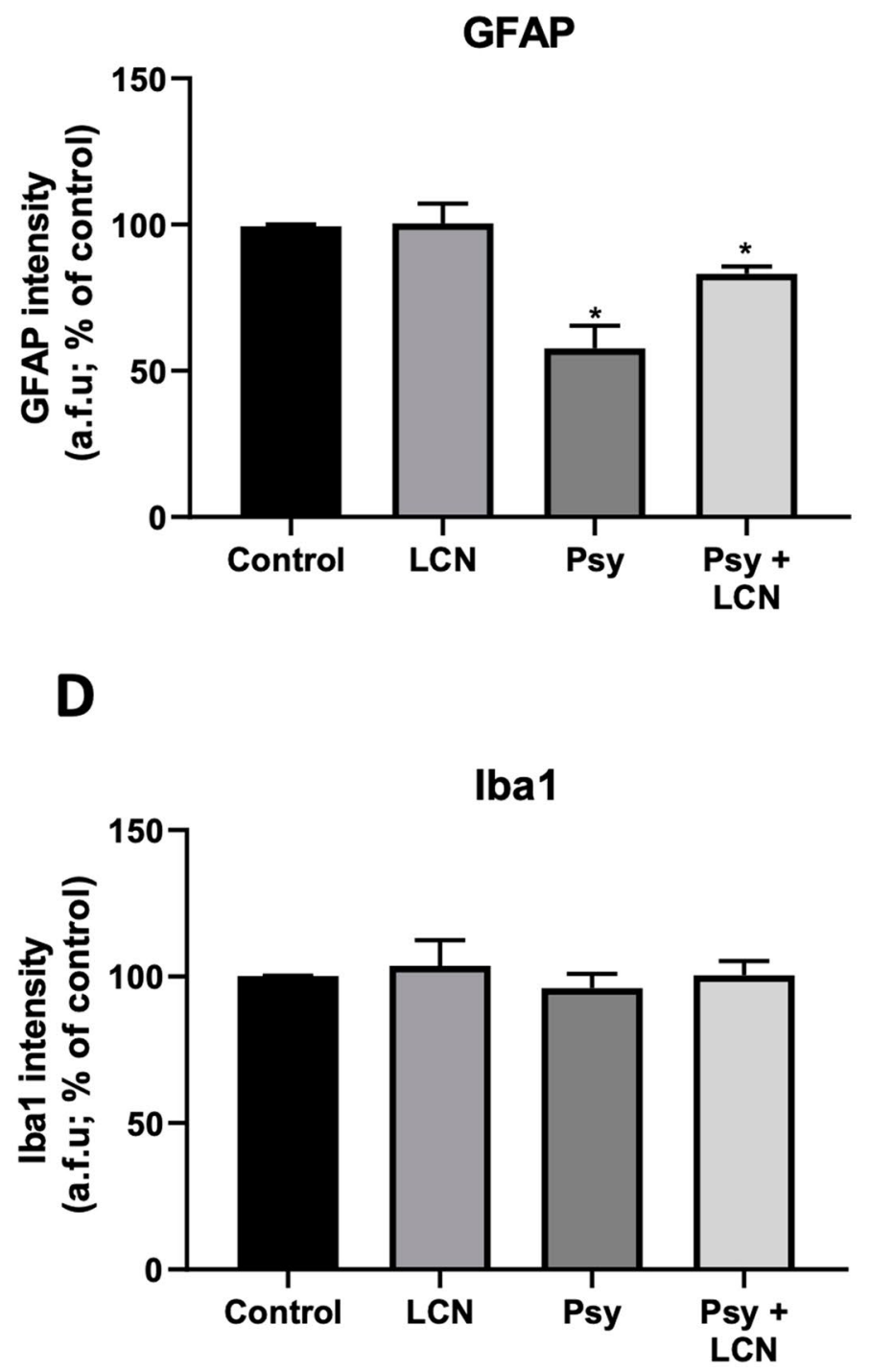

the fluorescence levels of vimentin. E, F Analysis of the microglial marker Iba-1 showed no effects on the levels of intensity or apparent morphology of microglia. Scale bars $=100 \mu \mathrm{m}$. Data shown as mean \pm SEM. Repeated measures one-way ANOVA following HolmSidak's post hoc test was performed. $N=6$

pharmacological approaches that can improve and cure these patients is urgent. One of the main challenges scientists face when developing new treatments for CNS pathologies is the $\mathrm{BBB}$. Thus, developing new therapeutic approaches that are able to bypass the BBB improving drug delivery to the brain is an ideal solution. Here, we report the potential use of hybrid lecithin/chitosan nanoparticles designed and already studied for nose-to-brain administration [14] in the dampening of psychosine and its protective effects against the cytotoxic effects of this toxin both, in vitro using human astrocytes and ex vivo using organotypic slice cultures. In this 
study, we have shown that these nanoparticles can prevent Psy-induced demyelination ex vivo, also preventing the associated axonal damage. Furthermore, despite that they failed to fully protect against the astrocytic death caused by Psy in this model, they led to lower cytotoxicity in astrocytes and early glia, in line with the full protection observed in vitro.

\section{Hybrid Lecithin/Chitosan Nanoparticles as a New Therapeutic Approach for CNS Pathologies}

As mentioned before, BBB separates the CNS from the systemic circulation protecting it from the entry of many pathogens and cells, but also being a challenge when delivering drugs to the CNS [7]. It is considered that the nose-to-brain administration route with nanoparticle-based delivery strategies could improve the cerebral bioavailability of many drugs $[24,25]$. Recently, our group has proposed that hybrid nanoparticles are composed of natural polysaccharides and phospholipids as an efficient system for drug delivery for various administration routes [14, 26, 27]. In this novel study, we proposed the lecithin/chitosan nanoparticles formed by spontaneous self-assembly not only as an efficient vehicle for drug delivery to the brain, but as a treatment on its own for the rare leukodystrophy Krabbe's disease model in vitro and ex vivo.

\section{LCN Protect from Psy-Induced Cytotoxicity Likely due to its Physicochemical Properties}

In the past years, astrocytes and their function have gained recognition as an important factor contributing to the onset and progression of many CNS pathologies [28], and it has been shown in multiple studies that they contribute to the neuroinflammatory state of the CNS, thus contributing to chronic and exacerbated inflammation in disease [18, 29]. Previous studies in our lab have also shown that Psy causes dysfunction in astrocytic function and is cytotoxic to this cell type, as well as showing that these effects could be closely linked to the pathogenesis of Krabbe's disease [4, 19, 30]. Here, we have shown that Psy induces morphological changes in astrocytes in vitro as observed by a reduction in the number of astrocytic projections, as well as cell death as measured by MTT assay (Fig. 1). Interestingly, we observed that the use of blank LCN not only demonstrated to be safe and well tolerated by human astrocytes (biocompatibility already observed for other human cell lines such as nasal RPMI2650 and monocytic THP-1 cells), but more importantly they were effective in protecting against the cytotoxic effects of Psy, without the action of any encapsulated drug. This supports the hypothesis that LCN structure is the responsible for this effect, probably correlated to the composition of nanoparticles formulation and due to its physicochemical properties. Psychosine, as a sphinganine glycosylated derivatives, has a pKa of 7.18. At acidic pHs ( 4.4),
Psy behaves as a mild soluble amphiphile forming micelles; however, at neutral environments, as in Krabbe's disease where Psy tends to accumulate in extralysosomal spaces, Psy is only partially charged. At pH 7.4, Psy micelles low surface charge may favor its organization into a more compact structure resulting in aggregates, as already observed by others [20]. This is in accordance to results presented here, where Psy alone was found by DLS to form structures with an average diameter of around $300 \mathrm{~nm}$ and a slightly positive surface charge $(\sim 10 \mathrm{mV})$. Psy micellar structures and larger agglomerates where evidenced in the cryoTEM images (Fig. 4A, B), even if the size of observed structures was significantly smaller compared to DLS data. These discrepancies are common when comparing DLS results with electron microscopy, since the DLS measures the hydrodynamic diameter of colloidal particles and provides an intensity weighed particle size distribution. Thus, the DLS measure is strongly affected by larger particles and agglomerates that scatter light with higher intensity and 'mask' the smaller particle scattering (see [31]). When evaluating the effect of the presence of Psy on the size, PDI and surface charge of lecithin/chitosan nanoparticles, we observed that the LCN increased in size while the electrical surface charge decreased, suggesting an interactions of the sphingolipid with the nanoparticles (Fig. 3). CryoTEM images disproved our initial and simplistic supposition that Psy structures were forming agglomerates with LCN nanoparticles by adsorbing on the nanoparticles surface. An alternative hypothesis could be that Psy, as a result of its tensioactive properties, is inducing a reorganization of the $\mathrm{LCN}$, possibly by coalescence of smaller particles.

This hypothesis is supported by some previous observations which evidenced that Psy $50 \mu \mathrm{M}$ toxicity on cultured primary fibroblasts from GLD patients was reduced by increasing concentrations of hydroxypropyl- $\beta$-cyclodextrin, a molecule able to complex hydrophobic molecules [32]. Similarly, Psy has been shown to integrate into phospholipid bilayers disturbing surface electrostatics and disordering the membrane hydrophobic portion [33]. In another study supporting the idea that Psy has mainly a membrane-based toxicity mechanism, it was highlighted that increasing concentrations of Psy $(5-50 \mu \mathrm{M})$ caused the swelling of DOPC liposomes in consequence of the sphingolipid partitioning in to the phospholipid bilayer [34]. These evidences appear particularly relevant for explaining the interaction of Psy with LCN nanoparticles, where phospholipids constitute the main component acting as surfactant for the lipid core and interacting electrostatically with chitosan. A perturbation in these functions, mediated by Psy integration in the nanoparticle structure, could cause particle instability leading to coalescence to reduce the surface area exposed to the water environment and favouring the attainment of a new equilibrium. Interestingly, however, no protective effect 
was observed for chitosan or lecithin alone, suggesting that nanoparticle components, as well as their specific organization (phospholipids surrounding a lipid core and stabilized by chitosan), play an important role for their efficient interaction with psychosine. These findings suggest that the interaction of Psy in LCN structure prevents the capacity of Psy to disrupt the astrocytic membrane and/or permeate the cells by physical sequestration, thus inhibiting its cytotoxic effects. How to exploit this scavenging action in a clinically relevant setting requires further investigation.

\section{Psy-Induced Demyelination and Axonal Damage is Prevented by LCN Treatment Ex Vivo}

As previously mentioned, Psy is a metabolic toxin that accumulates in the KD brains and is responsible for the oligodendrocyte death and extensive demyelination of both the central and peripheral nervous systems in pathology, leading to patients' death after few years of diagnosis [1, 2]. It has been demonstrated that the ex vivo model organotypic slice cultures are a viable method to study potential pharmacological treatments for KD or similar pathologies, as demyelination and Psy-associated effects can be mimic by adding Psy to this ex vivo system [4, 19]. Furthermore, cerebellar organotypic slice cultures provide a challenging environment of LCN, since the culture media will be rich in neurotransmitters, proteins and other substances secreted by cells $[30,35,36]$ and eventually competing or preventing Psy from interacting with LCN.

In this study, we have shown that treating organotypic slice cultures with LCN prevents the demyelination observed by decreased level of the myelin markers MBP and MOG (Fig. 5) and a prevention of the associated axonal damage, measured by expression of SMI-32 in the axons of the arbor vitae (Fig. 6). These results support our hypothesis that LCN are able to aggregate with Psy preventing its pathological effects, as observed in our ex vivo experiments.

When studying the effects of Psy and LCN on glial cells, we did not observe any change in microglia as measured by the microglia marker Iba-1 (Fig. 6). However, we note that this marker does not provide an insight into microglia reactivity, and therefore, further studies investigating the state of microglia would be required to analysed whether there is microglial activation under Psy conditions and if so, whether LCN are able to revert this effect. On the other hand, when analysing the astrocytic marker GFAP and early glia marker vimentin, we observed that Psy induce astrocytic death as measured by both a significant decrease in levels of GFAP and lower levels of vimentin, although the latter was not statistically significant (Fig. 6). These effects observed under Psy treatment are in line with what previous studies in our lab have shown [19, 22]. As expected, LCN also prevented astrocytic death induced by Psy, although it did not prevent it fully. However, LCN treatment prevented the astrocytic death to a similar extend as our in vitro studies in presence of Psy. This might be because of a higher sensitivity of astrocytes to the toxicity of Psy compared to other cell types as oligodendrocytes, likely due to different mechanisms of action of Psy depending on the cell type. We hypothesize that the protective effects of LCN against Psy are mainly due to physicochemical interactions which prevent Psy from exerting its toxic effects on the cellular systems. However, we also speculate the possibility of a competition between LCN and Psy for the astrocytic phospholipase A2 (PLA2). Previous studies in our lab have shown that activation of PLA2 by Psy leads to the formation of arachidonic acid metabolites, involved in inflammatory processes, oxidative stress and apoptosis in astrocytes [19]. In addition to this, we have also shown that lecithin, the main component of LCN, is a substrate of PLA2 [14, 26, 37]. Thus, a competitive inhibition of PLA2 by the LCN could also contribute to the prevention of Psy toxicity and might explain the differences in the extension of the protective effects of $\mathrm{LCN}$ in oligodendrocytes and astrocytes.

\section{Conclusion}

The data presented here demonstrate a novel and promising role of hybrid lecithin/chitosan nanoparticles in the treatment of demyelinating pathologies, specifically Krabbe's disease pathologies and those with an accumulation of toxins such as psychosine. Here, we report for the first time, the capacity of LCN to prevent Psy-induced glial cell death and demyelination both in vitro and ex vivo. We hypothesized that these effects are mainly relate to the physicochemical interactions of the LCN with the toxins, thus preventing Psy to exert its pathological effects. Therefore, this study gives evidence of the potential use of LCN not only as an efficient platform to delivery drugs to the CNS, as previously reported, but as a treatment itself in demyelinating pathologies such as Krabbe's disease.

Abbreviations AGS: Astrocyte growth factors; BBB: Blood brain barrier; BSA: Bovine serum albumin; CNS: Central nervous system; DIV: Day in vitro; DLS: Dynamic light scattering; DMEM: Dulbecco's Modified Eagle's Medium; DMSO: Dimethyl sulfoxide; $\mathrm{D}_{2} \mathrm{O}$ : Deuterated water; DOPC: 1,2-Dioleoyl-sn-glycero-3-phosphocholine; FBS: Foetal bovine serum; GALC: Galactosylceramidase; GFAP: Glial fibrillary acidic protein; HBSS: Hank's buffered salt solution; Iba1: Ionized calcium binding adaptor 1; IL: Interleukin; KD: Krabbe's disease; LCN: Lecithin/chitosan nanoparticles; MBP: Myelin basic protein; MOG: Myelin oligodendrocyte glycoprotein; NFH: Neurofilament H; n.s.: Non-significant; overnight: Overnight; P/S: Penicillin/ streptomycin; P10: Postnatal day 10; PALS: Phase analysis light Scattering; PBS: Phosphate-buffered saline; PLA2: Phospholipase A2; PDI: Polydispersity Index; Psy: Psychosine; RM: Repeated measures; RT: Room temperature; S1P: Sphingosine-1-phosphate; SEM: Standard error of the mean; TNF- $\alpha$ : Tumour necrosis factor-alpha; WMT: White matter tracts 
Supplementary Information The online version contains supplementary material available at https://doi.org/10.1007/s13311-021-01109-3.

Acknowledgements We would like to thank Ms Sibylle Bechet and $\mathrm{Mr}$ Luke Davison for technical help during this study. AC thanks the Brazilian National Council for Scientific and Technological Development$\mathrm{CNPq}$, as recipients of a grant in the program 'Ciências sem Fronteiras' [202558/2015-0].

Required Author Forms Disclosure forms provided by the authors are available with the online version of this article.

Author Contribution FS and KKD conceived the project and designed the research; MVE and AC performed the experiments; MVE and AC analysed the data; all authors discussed the data and results; MVE, AC, FS and KKD wrote the manuscript with contributions from all authors.

Funding Open access funding provided by Università degli Studi di Parma within the CRUI-CARE Agreement. This work was supported by an Irish Research Council PhD scholarship for M.V.E [GOIPG/2015/2804] and the Brazilian Government as CNPq GD-PhD scholarship and project grant to AC [202558/2015-0].

Open Access This article is licensed under a Creative Commons Attribution 4.0 International License, which permits use, sharing, adaptation, distribution and reproduction in any medium or format, as long as you give appropriate credit to the original author(s) and the source, provide a link to the Creative Commons licence, and indicate if changes were made. The images or other third party material in this article are included in the article's Creative Commons licence, unless indicated otherwise in a credit line to the material. If material is not included in the article's Creative Commons licence and your intended use is not permitted by statutory regulation or exceeds the permitted use, you will need to obtain permission directly from the copyright holder. To view a copy of this licence, visit http://creativecommons.org/licenses/by/4.0/.

\section{References}

1. Suzuki K. Globoid Cell Leukodystrophy (Krabbe's Disease): Update. J Child Neurol. 2003;18(9):595-603.

2. Giri S, Jatana M, Rattan R, Won J, Singh I, Singh AK. Galactosylsphingosine (psychosine) -induced expression of cytokine-mediated inducible nitric oxide synthases via AP-1 and C/EBP: implications for Krabbe disease. Faseb J. 2002;16(7):661-672.

3. Haq E, Giri S, Singh I, Singh AK. Molecular mechanism of psychosine-induced cell death in human oligodendrocyte cell line. J Neurochem. 2003;86(6):1428-1440.

4. O'Sullivan C, Dev KK. Galactosylsphingosine (psychosine)induced demyelination is attenuated by sphingosine 1-phosphate signalling. J Cell Sci. 2015;128(21):3878-3887.

5. Giri S, Khan M, Nath N, Singh I, Singh AK. The role of AMPK in psychosine mediated effects on oligodendrocytes and astrocytes: implication for Krabbe Disease. J Neurochem. 2008;105(5):1820-1833.

6. Mikulka CR, Sands MS. Treatment for Krabbe's disease: Finding the combination. J Neurosci Res. 2016;94(11):1126-1137.

7. Pardridge WM. The blood-brain barrier: bottleneck in brain drug development. Neurother. 2005;2(1):3-14.

8. Chen Y, Liu L. Modern methods for delivery of drugs across the blood-brain barrier. Adv Drug Deliver Rev. 2012;64(7):640-65.
9. Fortin D. Drug Delivery Technology to the CNS in the Treatment of Brain Tumors: The Sherbrooke Experience. Pharmaceutics. 2019;11(5):248.

10. Taki H, Kanazawa T, Akiyama F, Takashima Y, Okada H. Intranasal Delivery of Camptothecin-Loaded Tat-Modified Nanomicells for Treatment of Intracranial Brain Tumors. Pharmaceuticals. 2012;5(10):1092-1103.

11. Lorenzoni R, Davies S, Cordenonsi LM, Viçosa JA da S, Mezzomo $\mathrm{NJ}$, Oliveira AL de, et al. Lipid-core nanocapsules containing simvastatin improve the cognitive impairment induced by obesity and hypercholesterolemia in adult rats. Eur J Pharm Sci. 2020;151:105397.

12. Palazzo C, Laloy J, Delvigne A-S, Nys G, Fillet M, Dogne $\mathrm{J}-\mathrm{M}$, et al. Development of injectable liposomes and drug-incyclodextrin-in-liposome formulations encapsulating estetrol to prevent cerebral ischemia of premature babies. Eur J Pharm Sci. 2019;127:52-59.

13. Piazza J, Hoare T, Molinaro L, Terpstra K, Bhandari J, Selvaganapathy $\mathrm{PR}$, et al. Haloperidol-loaded intranasally administered lectin functionalized poly(ethylene glycol)-block-poly(d,l)-lactic-co-glycolic acid (PEG-PLGA) nanoparticles for the treatment of schizophrenia. Eur J Pharm Biopharm. 2014;87(1):30-9.

14. Clementino A, Batger M, Garrastazu G, Pozzoli M, Favero ED, Rondelli $\mathrm{V}$, et al. The nasal delivery of nanoencapsulated statins - an approach for brain delivery. Int J Nanomed. 2016; 11:6575-6590.

15. Clementino A, Sonvico F. Development and validation of a RPHPLC method for the simultaneous detection and quantification of simvastatin's isoforms and coenzyme Q10 in lecithin/chitosan nanoparticles. J Pharmaceut Biomed Anal. 2018;155:33-41.

16. O'Sullivan SA, O'Sullivan C, Healy LM, Dev KK, Sheridan GK. Sphingosine 1-phosphate receptors regulate TLR4-induced CXCL5 release from astrocytes and microglia. J Neurochem. 2018;144(6):736-747.

17. Rutkowska A, Preuss I, Gessier F, Sailer AW, Dev KK. EBI2 regulates intracellular signaling and migration in human astrocyte. Glia. 2014;63(2):341-51.

18. Elain G, Jeanneau K, Rutkowska A, Mir AK, Dev KK. The selective anti-IL17A monoclonal antibody secukinumab (AIN457) attenuates IL17A-induced levels of IL6 in human astrocytes. Glia. 2014;62(5):725-735.

19. Misslin C, Velasco-Estevez M, Albert M, O’Sullivan SA, Dev KK. Phospholipase A2 is involved in galactosylsphingosine-induced astrocyte toxicity, neuronal damage and demyelination. Plos One. 2017;12(11):e0187217.

20. Orfi L, Larive CK, LeVine SM. Physicochemical characterization of psychosine by $1 \mathrm{H}$ nuclear magnetic resonance and electron microscopy. Lipids. 1997;32(10):1035-1040.

21. O'Sullivan SA, Velasco-Estevez M, Dev KK. Demyelination induced by oxidative stress is regulated by sphingosine 1-phosphate receptors. Glia. 2017;65(7):1119-1136.

22. Velasco-Estevez M, Gadalla KKE, Liñan-Barba N, Cobb S, Dev KK, Sheridan GK. Inhibition of Piezo1 attenuates demyelination in the central nervous system. Glia. 2020;68(2):356-375.

23. Louis ED, Ma K, Babij R, Cortés E, Liem RK, Vonsattel J-PG, et al. Neurofilament protein levels: Quantitative analysis in essential tremor cerebellar cortex. Neurosci Lett. 2012;518(1):49-54.

24. Sonvico F, Clementino A, Buttini F, Colombo G, Pescina S, Guterres SS, et al. Surface-Modified Nanocarriers for Nose-to-Brain Delivery: From Bioadhesion to Targeting. Pharmaceutics. 2018;10(1):34.

25. Salem LH, El-Feky GS, Fahmy RH, Gazayerly ONE, Abdelbary A. Coated Lipidic Nanoparticles as a New Strategy for Enhancing Nose-to-Brain Delivery of a Hydrophilic Drug Molecule. J Pharm Sci. 2020;109(7):2237-2251.

26. Barbieri S, Buttini F, Rossi A, Bettini R, Colombo P, Ponchel G, et al. Ex vivo permeation of tamoxifen and its 4-OH metabolite through rat intestine from lecithin/chitosan nanoparticles. Int $\mathbf{J}$ Pharm. 2015;491(1-2):99-104. 
27. Şenyiğit T, Sonvico F, Rossi A, Tekmen I, Santi P, Colombo $\mathrm{P}$, et al. In Vivo Assessment of Clobetasol Propionate-Loaded Lecithin-Chitosan Nanoparticles for Skin Delivery. Int J Mol Sci. 2016;18(1):32.

28. Claycomb KI, Johnson KM, Winokur PN, Sacino AV, Crocker SJ. Astrocyte Regulation of CNS Inflammation and Remyelination. Brain Sci. 2013;3(3):1109-1127.

29. Sharma R, Fischer M-T, Bauer J, Felts PA, Smith KJ, Misu T, et al. Inflammation induced by innate immunity in the central nervous system leads to primary astrocyte dysfunction followed by demyelination. Acta Neuropathol. 2010;120(2):223-236.

30. O'Sullivan C, Schubart A, Mir AK, Dev KK. The dual S1PR1/ S1PR5 drug BAF312 (Siponimod) attenuates demyelination in organotypic slice cultures. J Neuroinflamm. 2016;13(1):31.

31. Bhattacharjee S. DLS and zeta potential - What they are and what they are not? J Control Release. 2016;235:337-351.

32. Salazar D, Jairo H, Fabian I, Bhardwaj K, Hanbali S, Rodrigues J, et al. Effects of cyclodextrin analogs on psychosine cytotoxicity. Mol Genet Metab. 2020;129(2):S142.

33. Díaz Y de las MZ, Caby S, Bongarzone ER, Fanani ML. Psychosine remodels model lipid membranes at neutral $\mathrm{pH}$. Biochim Biophys Acta. 2018;1860(12):2515-2526.
34. Hawkins-Salsbury JA, Parameswar AR, Jiang X, Schlesinger PH, Bongarzone E, Ory DS, et al. Psychosine, the cytotoxic sphingolipid that accumulates in globoid cell leukodystrophy, alters membrane architecture. J Lipid Res. 2013;54(12):3303-3311.

35. Huuskonen J, Suuronen T, Miettinen R, Groen T van, Salminen A. A refined in vitro model to study inflammatory responses in organotypic membrane culture of postnatal rat hippocampal slices. J Neuroinflamm. 2005;2(1):25.

36. Croft CL, Wade MA, Kurbatskaya K, Mastrandreas P, Hughes MM, Phillips EC, et al. Membrane association and release of wild-type and pathological tau from organotypic brain slice cultures. Cell Death Dis. 2017;8(3):e2671-e2671.

37. Barbieri S, Sonvico F, Como C, Colombo G, Zani F, Buttini $\mathrm{F}$, et al. Lecithin/chitosan controlled release nanopreparations of tamoxifen citrate: Loading, enzyme-trigger release and cell uptake. J Control Release. 2013;167(3):276-283.

Publisher's Note Springer Nature remains neutral with regard to jurisdictional claims in published maps and institutional affiliations. 